\title{
RIESGO DE HELADAS EN CULTIVOS. ANÁLISIS Y EVALUACIÓN
}

\author{
Alfredo Pérez Morales \\ Departamento de Geografía. Universidad de Murcia \\ A ti, que siempre me enseñas a ser buen Geógrafo y mejor persona
}

\section{RESUMEN}

Las heladas constituyen uno de los fenómenos atmosféricos que mayores daños económicos producen sobre la actividad agraria española. El campo murciano es especialmente sensible a este tipo de eventos debido a una serie de transformaciones en sus formas de producción a lo largo de las últimas décadas que han motivado un incremento de la vulnerabilidad a dicho peligro. Ante esta situación, se precisan estudios de localización como el presente, donde se identifican con detalle los principales sectores de riesgo para la posterior propuesta de medidas de actuación.

Palabras clave: Riesgo, probabilidad, exposición, helada, cartografía.

\section{ABSTRACT \\ Risk of frost in crops. Analysis and assessment}

Frost is one of the atmospheric phenomena that produce greater economic damage on the spanish agricultural activity. The Murcia field is particularly sensitive to such events due to a series of changes in their production methods over the past decades that have led to an increased vulnerability to that hazard. Under these circumstances, localization studies such as the present one are needed, in order to identify the main risk areas and then, proposing performance measures.

Keywords: Risk, probability, exposure, frost, cartography.

\section{INTRODUCCIÓN}

Las heladas son uno de los peligros de origen meteorológico que mayores daños económicos producen sobre los cultivos en el ámbito mediterráneo español, detrás de las sequías y las inundaciones (Olcina, 1995: 99-143). Se trata 
de eventos que han ido adquiriendo una severidad creciente, que se pone de manifiesto en una acentuación del volumen de pérdidas económicas asociadas a los efectos de este tipo de peligro. Al parecer, los registros térmicos mínimos absolutos de los predios rústicos, han dejado de ser vistos como condicionante natural desde la implantación de nuevas tecnologías de protección contra heladas pero, sobre todo, por la utilización de nuevas variedades extra tempranas y tardías. Éstas últimas hacen factible ampliar el calendario agrícola con lo que, bajo criterio del agricultor, se evitan los periodos potenciales de experimentar los efectos de las bajas temperaturas y se maximiza el rendimiento productivo y económico. Sin embargo, con esta forma de proceder, se aumenta a todas luces la exposición a eventos térmicos mínimos que acontezcan fuera del los meses propiamente invernales y, con ello, las citadas pérdidas y frecuencia de eventos.

El objetivo del presente trabajo es doble: De un lado, se analizarán todas las particularidades que atañen al peligro de helada y su influencia sobre la actividad agrícola en un ámbito que representa de forma paradigmática el conflicto antes señalado, la Región de Murcia. Una vez caracterizado el problema físico, estimar la probabilidad del riesgo de heladas con el fin de ayudar a los agricultores a decidir ¿Qué?, ¿Cuándo? y ¿Dónde? se planta (Snyder et al., 2010: 73-98). Para ello se elabora una cartografía que permite evidenciar la incidencia de dicha probabilidad del riesgo de heladas. Se trata de una primera aproximación de carácter integral, que representa una herramienta tanto para los agricultores como las aseguradoras. Resulta de fácil aplicación y de gran utilidad en la toma de decisiones reduciendo su grado de incertidumbre.

\section{CARACTERIZACIÓN DE LAS HELADAS AGROMETEOROLÓGICAS}

\section{Tipos de heladas}

En términos generales, el fenómeno meteorológico que provoca la helada tiene lugar cuando la condensación se produce a temperaturas inferiores a la de congelación, en cuyo caso la humedad pasa directamente del estado de vapor al de sólido o hielo. Para el caso que nos ocupa, es decir, las temperaturas mínimas a partir de las cuales afectan a los cultivos, la Organización Meteorológica Mundial define helada agrometeorológica en su base de datos terminológica METEOTERM como: «todo descenso térmico igual o inferior a $3{ }^{\circ} \mathrm{C}$ medido en el abrigo meteorológico, lo que equivaldría a $0{ }^{\circ} \mathrm{C}$ o menos a la intemperie en superficie». Por tanto, cuando se alcanza dicho umbral crítico, se producen las heladas que afectan a plantas, frutos y flores, cuyo grado de afección sobre los mismos, viene dado por la combinación de una serie de variables entre las que están: la duración de las bajas temperaturas, la humedad del aire, pérdidas por radiación solar directa, concentración de la savia, capacidad calorífica de la planta o fruto, grado de insolación y temperatura a la que empiezan a originarse efectos nocivos para el normal desarrollo de los cultivos. 
Espacialmente, se trata de un conjunto de procesos naturales que acontecen con mayor frecuencia en dominios climáticos subtropicales o climas influenciados por la altitud y disposición de los relieves en relación a los flujos dominantes de las masas de aire. Los primeros, que es donde se localiza la principal superficie del área de estudio, se caracterizan por presentar estaciones bien definidas, en las que el ritmo de las brotaciones y el desarrollo vegetal de los cultivos, están controlados por los cambios en los registros térmicos, siendo los del periodo invernal los más propicios a este tipo de fenómenos agrometeorológicos como son las heladas, pero sin olvidar, sobre todo, periodos de principios o tardo invernales, que a la postre, son los más dañinos para aquellos cultivos cuyo periodo vegetativo se adelanta artificialmente o por la presencia de frutos en invierno de los tropicales adaptados a algunas comarcas, caso de los cítricos.

De acuerdo a Olcina y Soriano (2002:631-633) se pueden distinguir dos situaciones que tienen al frío intenso como elemento atmosférico protagonista: a) los temporales de frío y nieve y b) las jornadas de helada fuerte. Las primeras están relacionadas con advecciones de masas de aire muy frías en invierno (polar continental o ártico marítimo), que provocan la precipitación de abundante nieve en buena parte del territorio peninsular. Estas olas de frío generan unos descensos muy acusados de las temperaturas cuyos efectos y tipos de helada dependerán del origen de la masa de aire. De producirse con aire cargado de humedad, en agroclimatología a esta clase de eventos se le llama comúnmente como «helada blanca». En esas ocasiones, de producirse una condensación sobre las plantas y objetos situados sobre la superficie y la temperatura desciende por debajo del punto de congelación del agua, el rocío depositado pasa a formar cristales de hielo y da lugar a la escarcha. En caso contrario, es decir, si la situación viene originada por la advección de una masa de aire frío de procedencia continental, la humedad atmosférica es baja y el punto de rocío puede hallarse por debajo de $\operatorname{los} 0^{\circ} \mathrm{C}$. Este tipo de heladas se conocen como «heladas negras», puesto que los vegetales muestran un ennegrecimiento de los órganos afectados (García y García, 1991).

Las segundas, o jornadas de helada fuerte, vienen asociadas a situaciones anticiclónicas, sobre todo aquellos días sin nubes y con viento en calma. Bajo esas circunstancias, la irradiación infrarroja nocturna provoca una pérdida de calor que se traduce en un enfriamiento del suelo y de las capas de aire en contacto con este, lo que provoca, en la mayoría de las ocasiones, el tipo de «helada negra» por inversión térmica y acumulación de frío intenso en las partes bajas bloqueado por el cálido de las capas superiores. Se trata de eventos muy temidos en las zonas de valle como la Huerta de Murcia y Vega Media del Segura, que los agricultores tratan de evitar rápidamente mediante la aplicación de las medidas protectoras activas y pasivas que se comentan en el siguiente apartado.

Por último, existe otro tipo de heladas de carácter combinado de las dinámicas atmosféricas antes señaladas (advección y estabilidad) que tienen por 
nombre, heladas de evaporación. En estas ocasiones, si por descenso de las temperaturas se ha originado condensación sobre las plantas, es decir, se reduce la humedad relativa del aire, al amanecer el incremento de temperatura provocado por la incidencia de rayos solares acentúa la evaporación rápida del rocío sobre la planta con el consiguiente enfriamiento debido a la cesión de calor latente. La intensidad de la helada dependerá de la temperatura del aire, de la cantidad de agua evaporada y, sobre todo, de la concurrencia de vientos de componente norte de origen continental con baja humedad relativa.

\section{Técnicas de defensa y métodos de protección}

En general, todos los métodos de protección contra heladas se basan en la prevención o la sustitución de la pérdida de calor. Obviamente, la elección adecuada de una u otra técnica dependerá de muchos factores según las características del lugar donde se apliquen. Sin embargo, a fin de establecer un orden de las mismas, se propone la siguiente clasificación de aquellas que con mayor o menor frecuencia se practican en el área de estudio adaptando el orden ya propuesto por Baeza (1993):

\section{- Métodos preventivos}

Análisis, registro, seguimiento y pronóstico de eventos, Aunque las temperaturas mínimas podrían variar en un ámbito de pronóstico debido a la presencia de microclimas, las condiciones meteorológicas predominantes para una explotación agraria suelen ser similares durante un episodio de helada. Es por tanto muy interesante conocer y registrar el comportamiento de cada evento en distintas zonas de un área, tratando de monitorizar temperaturas mínimas, nubosidad y la velocidad del viento.

Del mismo modo, el registro de series temporales es un factor esencial para predecir futuros escenarios y actuar en consecuencia. La información recopilada permite ubicar medidas de protección estructurales en lugares adecuados y maximizar su rendimiento. A modo de ejemplo, en situaciones de heladas de irradiación, el análisis detallado de los registros permite aumentar la eficiencia en la toma de decisiones sobre si iniciar actuaciones o no. Asimismo, disponer de series largas de información climática acerca de registros mínimos absolutos facilita la selección de especies y el tipo de equipamiento e infraestructuras a emplear para su protección.

Medición de la temperatura y humedad del aire. El control y seguimiento de la temperatura y humedad del aire ha sido desde siempre la medida de carácter preventivo más extendida en su empleo debido a lo reducido de su coste. En aquellas explotaciones que cuentan con cierto grado de innovación hacen uso 
de psicrómetros que, distribuidos por las parcelas de cultivo, permiten a los agricultores advertir la llegada de una helada por medio de un procedimiento de cálculo sencillo in situ.

Medición de la temperatura de la planta. Aunque es el menos practicado de todos los del grupo de los preventivos debido a su complejidad en el manejo e instalación, sus resultados suelen ser bastante efectivos en condiciones controladas. Por norma general, la temperatura de una cosecha (ya sea en forma de bulbo, flor y fruto) es menor que el aire circundante, lo que puede inducir fácilmente a error y no actuar como consecuencia de la confianza depositada en los registros aéreos cuando estos se encuentren por encima de los $3^{\circ} \mathrm{C}$. Sin embargo en la realidad, es posible que el cultivo empiece a experimentar los primeros efectos fitofenológicos debido a su temperatura más baja. Bajo estas circunstancias existe la posibilidad de completar la información para minimizar el grado de incertidumbre ante una decisión por medio de un instrumento conocido como termopar (también llamado termocupla). Se trata de un termómetro digital que dispone de sensores que, instalados en las partes que interese de la planta, proporcionan la información acerca de la temperatura interior de la misma, tan necesaria en situaciones de helada por advección de aire por el factor windchill.

- Métodos de protección pasiva

Elección de especiales y variedades resistentes, con una adaptación de los cultivos a los climas. Consiste en elegir especies y variedades que no tengan problemas de heladas en el área de puesta en cultivo. Hay que considerar la resistencia al frío de las diferentes éstas, y las diferencias fenonólogicas existentes, por ejemplo, en la fecha de floración de especies frutales.

Retraso de la época de floración, obtenido por medio del encalado de las yemas y ramas jóvenes. Es una medida de carácter convencional y rudimentaria que se aplica, principalmente, sobre los frutales a fin de salvaguardar las partes más vulnerables de la planta de los efectos del frío extremo.

Juiciosa elección de la situación de las parcelas. Se trata de ubicar la plantación en zonas de fácil drenaje del aire frío y solana, y evitar zonas de umbría deprimidas donde se acumule. También son zonas más propensas al frío las próximas a grandes masas de agua continental y a grandes extensiones de praderas o bosque.

Orientación de líneas de plantación en paralelo a setos y cortavientos. Se puede llegar a dificultar la entrada de aire frío en las parcelas mediante barreras a barlovento y también se evita que el aire se estacione sobre la parcela mediante la eliminación de obstáculos que impiden su salida. 
Poda alta y poda tardía. Se llevan a cabo para elevar la planta con respecto al suelo y alejarla de las capas más frías (ej. Cultivos emparrados de uva) y para retardar la brotación de partes de la planta vulnerables.

Enterramiento temporal de la planta. En ocasiones extremas, para el caso de la huerta tradicional, se procedía al enterramiento de la planta a fin de protegerla y conservar su temperatura interna bajo tierra.

Abonados. La enmienda de materia orgánica en descomposición avanzada permite la liberación de calor por medio de irradiación durante ese proceso lo que compensa la pérdida de calor de la planta y el calentamiento por convección del aire circulante en contacto con dichos abonos.

Eliminación de malas hierbas. La presencia de vegetación en el suelo aumenta la superficie de irradiación y las pérdidas de calor durante la noche. Así, en plantaciones de frutales de la vega media del río Segura, el procedimiento que se sigue es el de eliminar mediante laboreo superficial y no labrar hasta que el riesgo de heladas se haya mitigado.

Seguro de pérdidas por helada. Es un contrato entre un asegurado (agricultor) y un asegurador (Compañía de Seguros), que abona un indemnización por daños. Al fijar la cuantía de las primas de dinero se tienen presentes los riesgos específicos (tipo de cultivos, estado de desarrollo) y los riesgos topográficos y climáticos (duración del periodo de heladas, frecuencia e intensidad de las mismas).

Si tiene lugar la helada, después de ésta se lleva a cabo una peritación para fijar la cantidad a indemnizar. Así, pues, el seguro contra la helada es una protección pasiva, basado en la cooperación y solidaridad de los agriculturos, que cuenta con el apoyo y respaldo del Estado a través del Consorcio de Compensación. Actualmente se promocionan varios tipos de seguros combinados para el campo: heladas-predisco-sequía-viento-incendio-inundación.

- Métodos de protección activa

Estrategias de riego. El conocido más comúnmente es el riego por aspersión. El calor que pierde la planta por irradiación es sustituido por el calor que libera el agua cuando inicia su proceso de congelación. En concreto, la relación es de 1 gr de agua congelada/80 calorías. El riego por inundación en momentos de helada evita de esta forma el enfriamiento de las capas de aire en contacto con el suelo dificultando su acumulación y la congelación de raíces a través de poros y macroporos del suelo. Además, de producirse la evaporación, condensa sobre las partes altas de la planta lo que aumenta la protección. 
Formación de pantallas aéreas artificiales. La finalidad de este método es el de acrecentar el efecto invernadero y atenuar la pérdida de calor. En casos como el área de estudio, los agricultores producen dicha nube por medio de la combustión de material orgánico inflamable procedente de las podas realizadas a las plantas y árboles, pacas de paja o forraje e, incluso, neumáticos o plásticos (estos últimos, obviamente, totalmente prohibidos debido a la liberalización de gases nocivos a la atmósfera). Con ese procedimiento se eleva la temperatura de las partes bajas y se produce una nube de humo que, en situaciones de inversión térmica por estabilidad atmosférica, se estabiliza por encima de la parcela de cultivo y ejerce de pantalla reflectora del calor liberado por las plantas a la atmósfera.

Mecanismos de viento. El principal propósito de este método es el de favorecer la agitación de la atmósfera (mezcla del aire superior con el inferior) por medio de mecanismos que faciliten la circulación del aire como ventiladores e, incluso, helicópteros. Aunque este tipo de técnicas solo son efectivas bajo circunstancias de heladas de irradiación, los ventiladores suelen ser bastante eficientes para superficies de alrededor de las 4 hectáreas con máquinas de un diámetro de 5 metros montadas sobre torres de 10 metros y, además, consumen entre un $5-10 \%$ de la que usan los calentadores convencionales.

Calentadores. El calentamiento ha sido utilizado durante siglos como medida de protección por medio de quemas de materiales fácilmente inflamables. Sin embargo, desde que la combustión comenzó a realizarse por medio de hidrocarburos, debido al encarecimiento de estos últimos, este tipo de técnicas ha tratado de buscar otro tipo de combustibles sustitutivos como: hueso de frutal, cáscara de almendra y pellets de madera, principalmente. La gran ventaja de esta técnica con respecto a las anteriores es que posibilita a los agricultores el control de la tasa de combustión para el control de la temperatura de la parcela de cultivo. Los calentadores proporcionan protección por medio de dos tipos de mecanismos: De un lado, el aire caliente emitido permite atemperar por convección el aire circundante a los cultivos, y de otro, la propia irradiación de la caldera o dispositivo calentador, siempre y cuando éste se encuentre situado en un lugar de la explotación, y que dicho aporte energético sea aprovechable por las plantas. En el área de estudio, este tipo de técnicas se emplean en la actualidad de forma eficienciente en superficies de invernaderos que estén altamente tecnificadas o de última generación.

Cubiertas artificiales. Éste método consiste en cubrir el suelo con sustancias de escaso poder radiante (plástico). Cuando el tipo de cultivo es de reducidas dimensiones, como así sucede con las propias de la hortofruticultura de ciclo manipulado, se tapan por medio de acolchados o materiales plásticos individualizados por planta. La versión de mayor dimensión de este tipo de técnica es el 
invernadero, el cual, como ya se ha mencionado anteriormente, suele combinarse con mecanismos de calentamiento artificial.

Métodos bioquímicos. Son sustancias que permanecen todavía en estado experimental y que tienen como finalidad la modificación del punto de congelación de la planta o tratando de inhibir la formación de escarcha y hielo sobre la misma.

\section{LA EVOLUCIÓN DEL RIESGO DE HELADA EN EL ÁREA DE ESTUDIO}

Sin duda alguna, la temperatura se convierte en un factor físico que, junto a la precipitación, la insolación y la luminosidad, son definidores del tipo de agricultura que se viene practicando en cada zona. Su análisis permite establecer diferencias y matices entre las regiones españolas donde se realiza una agricultura que, al referirse a la del sureste de la Península Ibérica, demuestra ser una de las que han experimentado mayores cambios y más dinamismo en las últimas décadas, lo que, como se señala más adelante, puede haber supuesto una asunción del riesgo de heladas por parte de sus practicantes.

Sin entrar en el debate sobre la posible contribución que haya podido tener el factor físico por encima del humano en lo que se refiere al aumento de este tipo de episodios naturales (ver Fernández-Long et al., 2013: 1673-1686), parece que, a la vista de la frecuencia de heladas acontecidas en las últimas décadas en el área estudiada (La Región de Murcia) (CAGP-CEBAS 1992; Fernández et al., 2003: 95), existe una aptitud por parte de los agricultores negligente, en la que no se considera la posibilidad de que las heladas puedan incidir de forma negativa sobre la hortofruticultura y confiada en la actitud solidaria de las medidas aseguradoras.

Las heladas en el campo de Murcia presentan una significación creciente que se manifiesta tanto en su frecuencia, debido a un incremento de las zonas cultivadas expuestas al peligro, como en lo que se refiere al valor económico de las pérdidas, por el aumento en cuanto al valor de los cultivos que se producen. Ese desajuste entre los funcionamientos del sistema agrícola y el ambiental, viene explicado por un proceso expansionista y de cambio intenso en los sistemas agrícolas durante los últimos sesenta años. Desde 1953, se empezó a contar con nuevos recursos hídricos procedentes de la construcción del gran embalse de cabecera, el Cenajo, y una serie de ayudas que se dotaron a las comarcas de Campo de Cartagena, Vega Alta y Bajo Guadalentín, para la captación de aguas subterráneas. Esta tendencia expansionista de la superficie cultivable y regable se vio finalmente consolidada con la llegada de las primeras aguas del Tajo (1980), las cuales permitieron el incremento del regadío hasta 1993, año en el que se alcanzó la mayor área de superficie regada en la Región de Murcia, con unas 193.000 ha. (Morales, 2001). Así mismo, la demanda de frutas y hortalizas, que desde los mercados de la UE se vio intensificada desde 1986, obligó 
a los agricultores murcianos a intentar responder a ella para beneficiarse de los buenos márgenes comerciales proporcionados por las ventas en dichos mercados. Esta espectacular transformación del regadío murciano, es la que va a dar lugar a la existencia en 2004 de unas 54.535 ha. de regadíos hortícolas y unas 59.844 de frutales de hueso y uva, todos ellos de alto valor comercial junto a unas 15.000 ha. de cítricos modernizados que van a constituir las 130.000 ha. básicas productivas del regadío murciano (Pérez, 2006). A esta última cifra hay que añadirle las algo más de 400.000 ha de secano para estimar una superficie cultivable expuesta al peligro de unas 500.000 ha.

La tendencia anteriormente descrita, resulta muchas veces incompatible con la consecución de un modelo de ocupación adaptado a las características climáticas de una zona que, es de sobra sabido, a veces, se manifiesta de forma severa e inconciliable con un sistema de cultivos cada vez más vulnerable y que termina saldándose con un balance de pérdidas creciente. Dentro de las causas más destacables que justifican lo anterior se podrían señalar:

- Gracias a los avances tecnológicos experimentados en ese periodo, se favorece la roturación de sectores donde la frecuencia de heladas era manifiesta y que hasta entonces habían estado desocupadas para su aprovechamiento agrario, o si lo estaban, lo hacían con especies vegetales cuyo ciclo biológico estaba perfectamente adaptado al contexto ambiental dominante.

- Las heladas que no afectaban a masas vegetales anteriores (especies mejores adaptadas a las condiciones ambientales), ahora pasan a ser un problema desde el momento en que se seleccionan nuevos cultivos más sensibles a estas inversiones de la temperatura pero, por supuesto, de mayor valor comercial, siempre que se adelanta su floración a la segunda quincena de enero o primera de febrero. La intencionalidad es evidente, es decir, principalmente economicista (Morales, 2014: 631-652). Como ya se ha mencionado, la acentuación que se produce de dicho valor de la producción en el sureste peninsular en momentos invernales pero, principalmente, tardoinvernales, al ser uno de los únicos ámbitos europeos capaz de cosechar en ese periodo, se deriva en fuertes márgenes comerciales y despierta el interés entre los agricultores para maximizar el rendimiento de sus predios a pesar del peligro inminente de la helada.

Este conflicto ha derivado en un aumento sustancial de la exposición y respalda los objetivos del presente trabajo. Es decir, por un lado, conviene considerar y evaluar dicha exposición a fin de obtener conclusiones sobre los ámbitos en los que convendría actuar de forma más eficiente $\mathrm{o}$, al menos, establecer estrategias de cultivo o planificación agrícolas más adaptadas a las condicionantes ambientales. Asimismo, también se desarrolla un trabajo de cartografía del riesgo que, 
a todas luces, es de gran utilidad para reducir el grado de incertidumbre y percepción que influyen en los procesos de toma de decisión que competen a los agricultores para llevar a cabo sus explotaciones agrícolas.

\section{METODOLOGÍA Y FUENTES PARA LA ELABORACIÓN DE CARTOGRAFÍA DE RIESGO DE HELADA}

Para la realización de la cartografía de riesgo de helada se consideraron los dos componentes que motivan la presencia del mismo, el factor físico y el factor humano. El primero se abordó mediante un modelo probabilístico obtenido a partir de los registros térmicos de las 45 estaciones climáticas del Red del Sistema de Información Agrario de Murcia (SIAM). A fin de cubrir los vacíos o lagunas de la red anterior y tener una cobertura suficiente para llevar a cabo las posteriores interpolaciones de información, se completaron los registros con los datos de 15 estaciones del Sistema de Información Agroclimática para el Regadío (SIAR) pertenecientes al MAGRAMA (Ministerio de Agricultura, Alimentación y Medio Ambiente).

Dentro de los distintos modelos que se emplean para medir la probabilidad de helada, se optó por el de Poisson, al tratarse de un fenómeno que en el área de estudio no resulta tan frecuente como pueda suceder en otros ámbitos del interior peninsular o grandes altitudes. La función de densidad del mismo es:

$$
f(x)=P(X=x)=\frac{\lambda^{x} e^{-\lambda}}{x !}
$$

Donde $\lambda$ sería el número de heladas producidas por año en función del periodo de observación; e, la base de los logaritmos neperianos y x el número absoluto de meses con heladas (Raso et al., 1987: 273). Para este cálculo se consideró el umbral de los $0^{\circ} \mathrm{C}$ debido a que es el valor medio más aproximado a los umbrales de temperatura crítica en los que se desagrega la información espacial de cultivos empleada para evaluar la exposición.

Seguidamente, se cartografiaron los resultados interpolando los valores de probabilidad resultantes de cada estación y mes con presencia de heladas (diciembre-abril) mediante el método SPLINES, con un valor de tensión $60 \mathrm{y}$ sin ningún peso de ponderación tal y como indica Alonso (2004) y Quiñonero y Alonso (2007) (ver figura 1). Los resultados son mapas en formato raster de probabilidad de helada para cada mes considerado con una resolución de 1000 $\mathrm{m}$. En adelante se optó por continuar la confección de la cartografía final en formato vectorial debido incompatibilidades escalares con respecto al resto de información interviniente. Por ello, el mapa de peligro o probabilidad de helada se reclasificó en 5 grandes grupos (Muy Alta, Alta, Media, Baja y Muy Baja) mediante el método de intervalos iguales que, posteriormente, se vectorizaron 
y dieron como resultado un mapa de isolíneas con los mismos niveles de probabilidad.

En cuanto al factor humano, éste quedó representado, en lo que concierne a la exposición, mediante la nomenclatura de cultivos contenida en cobertura cartográfica del SIGPAC (Abril 2013). La identificación de dicha exposición se efectuó, en primer lugar, mediante una simple selección por atributos de aquellos usos que representan algún tipo de cultivo agrícola y, en segundo, una selección dinámica de la primera según los momentos de floración y fruto de cada uno de esos grupos de cultivos para cada mes con heladas. El proceso de identificación y filtrado anterior se puede resumir de acuerdo a lo recogido en la matriz que representa la tabla 1 . Donde «0», supone que no hay exposición, «1»

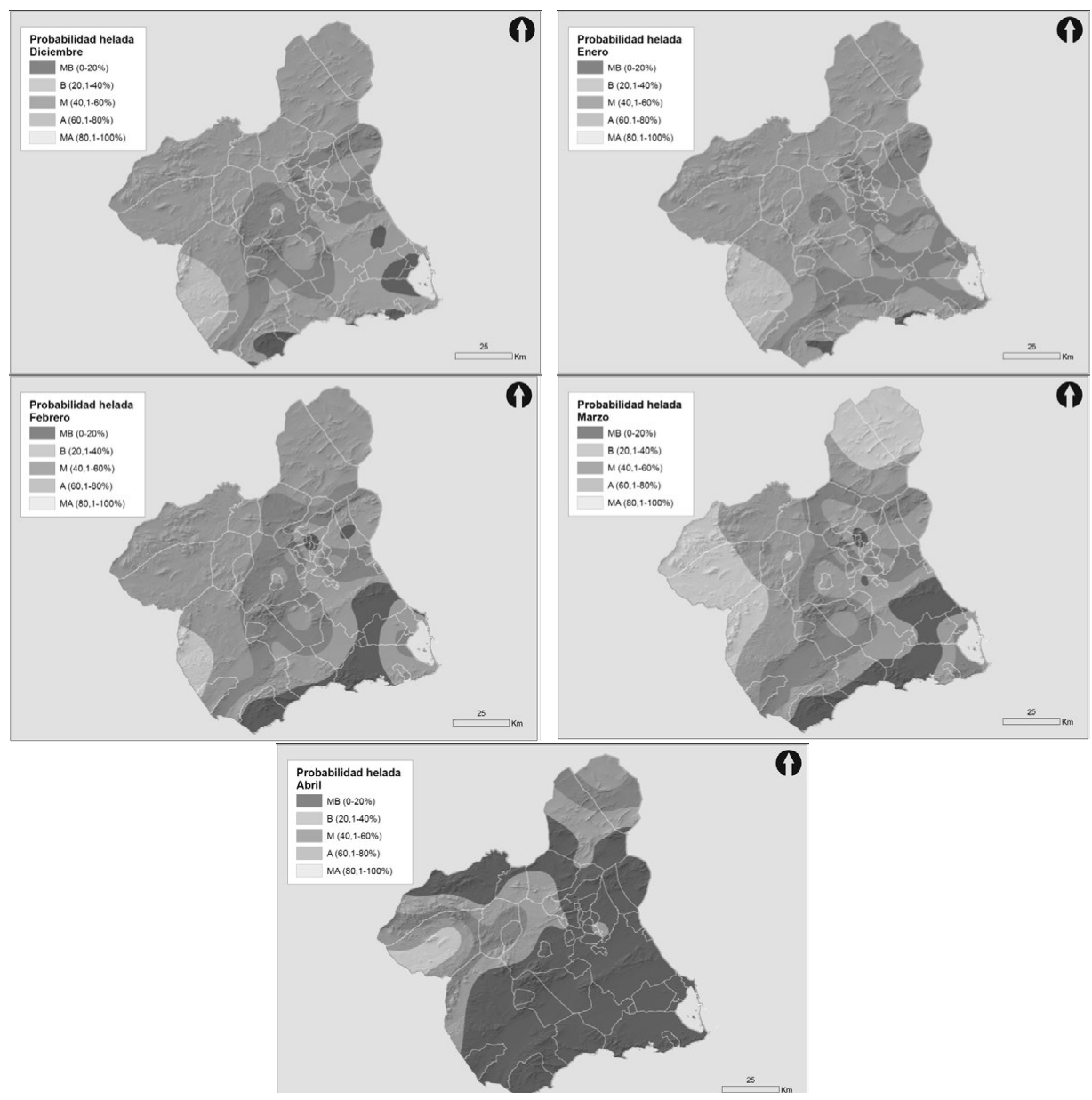

Figura 1. Mapas de probabilidad de helada para los meses de Diciembre a Abril 
es la exposición del fruto en ese mes y «2» la exposición durante el periodo de floración. El resultado de los pasos anteriores es un mapa de exposición según el mes de probabilidad de helada y el cultivo expuesto.

\begin{tabular}{|l|c|c|c|c|c|}
\hline Código de uso SIGPAC & Diciembre & Enero & Febrero & Marzo & Abril \\
\hline CF (Cítricos-Frutales) & 1 & 1 & 0 & 2 & 2 \\
\hline CI (Cítricos) & 1 & 1 & 0 & 0 & 2 \\
\hline $\begin{array}{l}\text { Cs (Asociación cítricos-Frutales } \\
\text { cáscara) Secano }\end{array}$ & 1 & 1 & 0 & 2 & 2 \\
\hline FF (Frutales-Frutales cáscaras) & 1 & 0 & 2 & 2 & 1 \\
\hline FL (Frutos secos y olivar & 1 & 0 & 0 & 2 & 2 \\
\hline FS (Frutos secos) & 0 & 0 & 2 & 0 & 0 \\
\hline FV (Frutos secos y viñedo) & 0 & 2 & 2 & 0 & 0 \\
\hline FY (Frutales) & 1 & 0 & 2 & 2 & 1 \\
\hline OC (Asociación olivar cítricos) & 1 & 1 & 0 & 0 & 2 \\
\hline OF (Olivar-Frutal) & 1 & 1 & 0 & 2 & 2 \\
\hline OV (Olivar) & 1 & 1 & 0 & 2 & 2 \\
\hline TH (Huerta) & 1 & 1 & 2 & 1 & 1 \\
\hline VF (Viñedo-frutal) & 1 & 0 & 0 & 2 & 2 \\
\hline VI (Viñedo) & 0 & 0 & 0 & 0 & 2 \\
\hline VO (Viñedo-Olivar) & 1 & 1 & 0 & 2 & 2 \\
\hline TA (Tierras arables) & 0 & 1 & 1 & 1 & 1 \\
\hline
\end{tabular}

Tabla 1. Exposición de cultivos según meses y usos del SIGPAC.

La labor metodológica culmina con la realización del mapa de riesgo a partir de la intersección de las dos cartografías anteriormente descritas, probabilidad de heladas y exposición. Esta representación gráfica nos permite identificar y clasificar las parcelas de cultivo del SIGPAC en cada uno de los grupos de riesgo según su probabilidad de materialización en forma de helada. Todo el procedimiento comentado se puede ver resumido en el modelo simplificado contenido en la figura 2.

\section{Resultados}

El procesamiento y análisis estadístico nos permite sintetizar unos resultados multiescalares de gran valor para estimar las superficies susceptibles de ser afectadas por la severidad de las temperaturas mínimas absolutas. Como se puede advertir en la figura 3, las comarcas del Noroeste, Altiplano Jumilla-Yecla y Campo de Lorca registran la mayor superficie cultivada expuesta a las heladas. 


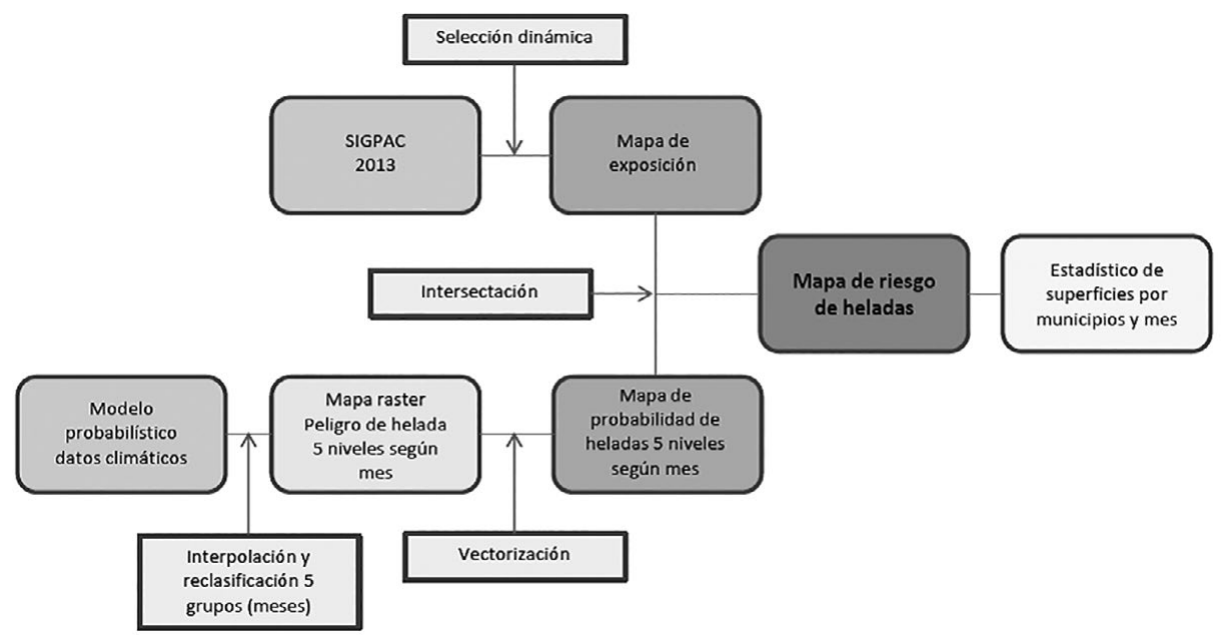

Figura 2. Modelo simplificado para la elaboración del mapa de riesgo de heladas en cultivos

En ellas, la frecuencia de las temperaturas por debajo de $\operatorname{los} 0^{\circ} \mathrm{C}$ es mayor debido a la elevada altitud media de sus territorios y, sobre todo, por la acentuación de los rasgos de continentalidad e inversión térmica nocturna en los valles corredores de estas comarcas de direcciones NE-SW. Esta condición de vulnerabilidad biofísica o natural del territorio supone un factor que, combinado con el incremento experimentado de cultivos poco adaptados a unas condiciones de mayores limitaciones térmicas en las últimas décadas, eleva el valor del riesgo por encima del resto del área de estudio. Por el contrario, las comarcas como el Campo de Cartagena, Comarca de Abanilla-Fortuna y Área Metropolitana de Murcia, aunque también han seguido un comportamiento algo similar a las anteriores, ostentan más del $75 \%$ de su superficie cultivada en zonas expuestas a una baja y muy baja probabilidad. Se trata de una cifra que garantiza y justifica estas prácticas agrícolas, las cuales, como ya se ha comentado, son más sensibles a las situaciones de descenso acusado de la temperatura diaria. En otras palabras, la menor vulnerabilidad biofísica de dichas comarcas minimiza sustancialmente el incremento del riesgo que pueda suponer el peligro. De esta forma, las contingencias producidas por las heladas, cuando se den, en gran parte se pueden llegar a salvar con medidas de protección ya comentadas.

En términos absolutos (ver figura 4), la cuantía superficial de los cultivos expuestos reflejan lo comentado en el apartado anterior. Destaca sobre todo el gran valor en hectáreas que se alcanza en las comarcas más afectadas por las heladas (Altiplano, Noroeste y Comarca de Lorca), que suman entre todas ellas una superficie potencialmente afectable, para todos los meses considerados de 560.500 ha. en las categorías de alta y muy alta probabilidad, es decir, el 37,5\% 


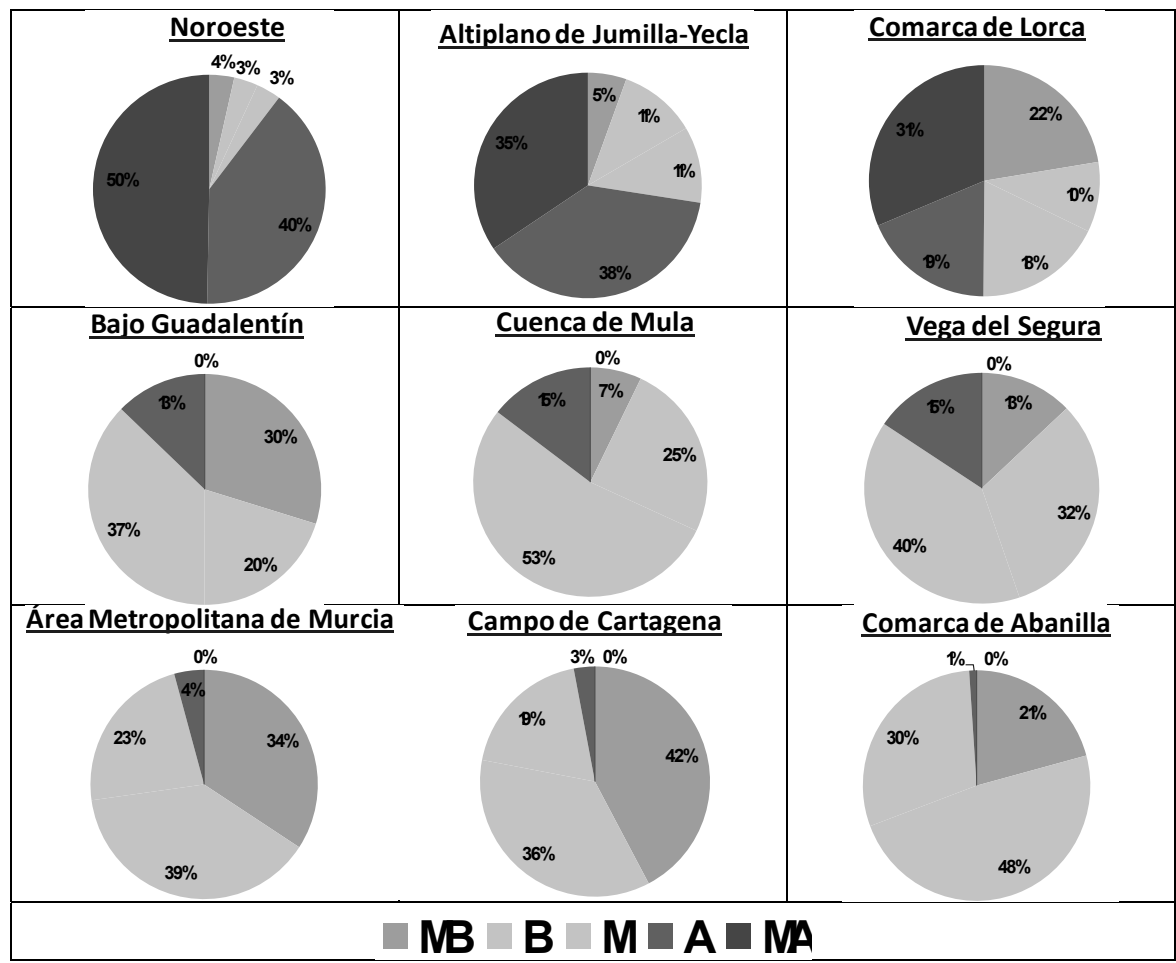

Figura 3. Porcentajes de superficie expuesta según distintos niveles de probabilidad de helada y comarcas.

del total expuesto, el 44\% de la superficie cultivable en esas comarcas y el 22,8\% para toda la Región. En el extremo opuesto se incluyen las comarcas donde la superficie de cultivos expuestos a heladas de alta y muy alta probabilidad de ocurrencia es muy reducida. Al igual que antes, estos son los casos de la cuenca de Abanilla, Área Metropolitana de Murcia y Campo de Cartagena. Entre todas representan una superficie cultivada expuesta de 24.702,34 ha en probabilidades altas y muy altas que son el $1,6 \%$ con respecto a total de lo expuesto, $3,8 \%$ de la superficie cultivable en esas comarcas y 1,0\% con respecto a toda la Región de Murcia.

Finalmente, todo lo comentado hasta el momento se evidencia de forma más clara en los resultados recogidos en la figura 5. En ésta aparecen recogidos los valores relativos acumulados mensuales de las superficies cultivadas expuestas a la probabilidad de heladas alta y muy alta con respecto al total cultivable de cada comarca. De la lectura de la misma se pueden derivar una serie de cuestiones: en primer lugar, se confirman las comarcas del Altiplano, Lorca y 


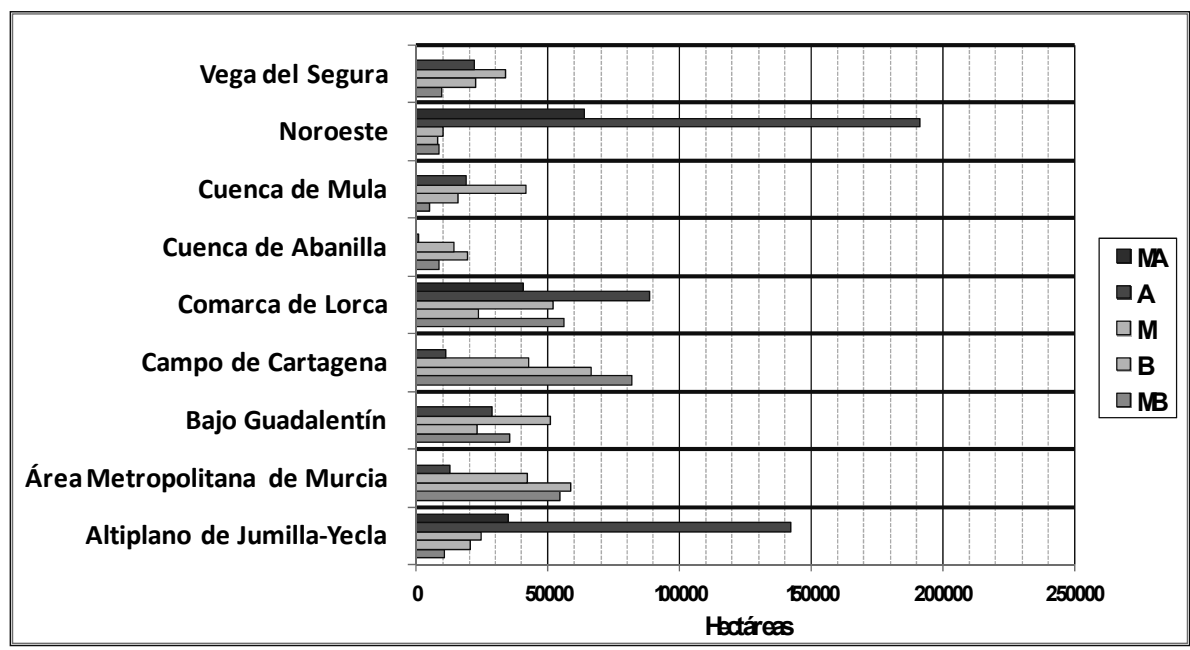

Figura 4. Valores absolutos de la superficie expuesta al peligro de heladas por comarcas.

Noroeste como las que poseen una mayor superficie expuesta y potencialmente afectable con todos los problemas que eso puede suponer en forma de pérdidas económicas. En segundo lugar, se aprecia como el mes de Enero es un momento verdaderamente crítico en el que el riesgo se generaliza de forma significativa por el territorio regional. Por último, las heladas tardías de abril en las probabilidades señaladas solo se advierten en las comarcas con los registros térmicos mínimos más rigurosos.

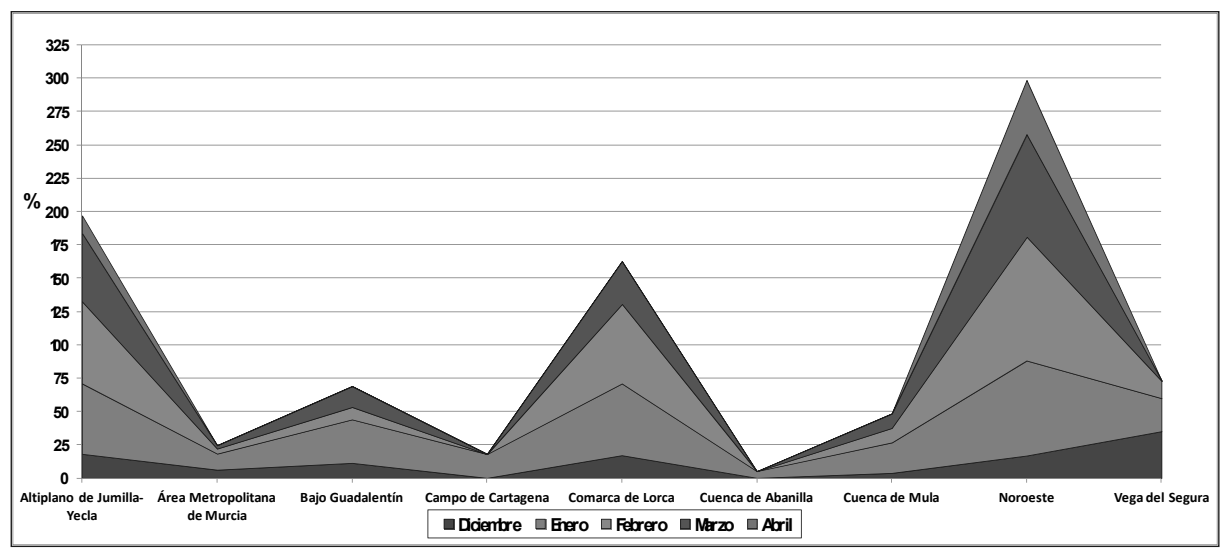

Figura 5. Valores relativos acumulados de las superficies expuestas al peligro de helada con probabilidad alta y muy alta por meses con respecto al total cultivable. 


\section{CONCLUSIONES}

En términos generales, los resultados cartográficos obtenidos evidencian una distribución espacial del peligro de helada que confirma la percepción cotidiana de este tipo de eventos y que, por tanto, se ajusta a la realidad en cada uno de los meses considerados. Se podría decir que, gracias a la disponibilidad de este tipo de herramientas y fuentes, ya se pueden ir aventurando trabajos de grandes dimensiones en cuanto a la información movilizada, como es el caso del presente, en el cual se han llegado a evaluar un total de 1,1 millones de parcelas de cultivo.

Pese a las bondades comentadas, existen algunas carencias que merecen ser señaladas. En lo que se refiere a su aplicabilidad, todavía no es apropiado realizarlo a escala de gran detalle debido, principalmente, a que el grado de cobertura de la red de estaciones meteorológicas con datos utilizables de libre acceso es bastante limitada y tiene escasa extensión temporal. La mayor disponibilidad de este tipo de registros permitiría analizar también: el tipo de heladas, diferenciando umbrales térmicos críticos; duración de horas de la temperatura extrema, condiciones de humedad, etc... cuestiones que hoy por hoy, sólo se pueden aplicar a lugares concretos y periodos recientes. Evidentemente, lo anterior es imprescindible para poder afinar el grado de resolución espacial en el mapa de peligro. De sobra es conocido (Olcina, 1995: 99-143) que el fenómeno de las heladas requiere de este tipo de detalle ya que su materialización en ciertos lugares, como es el caso de las parcelas de cultivo, viene muchas veces explicado por la disposición de los mismos con respecto a barlovento o sotavento de la advección de flujos del primer y segundo cuadrante, así como su localización en espacios deprimidos concretos donde se acumulan masas de aire frío en situaciones de estabilidad atmosférica.

Por su parte, la cartografía del componente humano de ese binomio que conforma el riesgo de helada, ha mejorado enormemente con la disponibilidad de fuentes de actualización periódica y perfil eminentemente agrario como es el SIGPAC. El grado de detalle al que llega dicha fuente, facilita su utilización en este tipo de trabajos gracias, sobre todo, a la cobertura y clasificación por usos agrícolas que contiene en su base de datos. Desafortunadamente, el grado de desagregación por cultivos no es todavía lo suficientemente preciso para desglosar los mismos según las características individuales que configuran cada uso. Lo anterior resulta un verdadero condicionante que debe ser superado a fin de poder definir con mayor detalle el grado de exposición al peligro en trabajos como el del riesgo de heladas, o incluso, riesgo de sequía, demandas hídricas, etc.

En lo que respecta a los resultados estadísticos obtenidos, se pueden diferenciar claramente dos ámbitos geográficos en función de la importancia del riesgo de heladas sobre ellos. Por un lado, las comarcas menos propicias para la 
implantación de una agricultura intensiva con amplio calendario agrícola serían: Altiplano, Noroeste y Lorca. En éstas cabría replantearse la idoneidad de la agricultura que se práctica en la actualidad, la cual, se encuentra en gran parte, escasamente adaptada a los condicionantes térmicos que originan daños más que probables y que, posiblemente, no sean compensados con los beneficios de años de bonanza sin helada. De otro lado, estarían las comarcas del Campo de Cartagena, Cuenca de Abanilla y Vega del Segura, donde la probabilidad de grandes pérdidas para este tipo de actividad, a pesar de existir, no supone un riesgo de largo recorrido que, traducido en pérdidas, haga necesario replantearse la actividad desde el punto de vista meramente agrícola y economicista.

La principal explicación del aumento de la exposición podemos buscarla en un efecto sinérgico de la vulnerabilidad biofísica (elevada altitud, continentalidad, disposición del relieve a los vientos dominantes) de ciertos ámbitos y el comportamiento negligente, carente de percepción e inducido por el interés económico de rentabilizar los terrazgos de espacios muy vulnerables. Los resultados estadísticos evidencian de forma meridiana esta relación desafortunada hombre medio, de forma que las comarcas más afectadas por las heladas, ostentan ya una superficie afectable del $44 \%$ con respecto al total de lo cultivable en área de probabilidad alta y muy alta. Por el contrario el Campo de Cartagena, Abanilla y Vega del Segura, apenas el 3,8\%. En definitiva, una superficie agrícola que por su ubicación, está bien justificada y se adecúa a las condiciones climáticas predominantes, es decir, temperaturas absolutas de los meses de invierno por encima de $\operatorname{los} 0^{\circ} \mathrm{C}$, insolación y luminosidad. Se trata de una conclusión que ya en su día Morales Gil (1997: 167) señalaba de forma sobresaliente en su trabajo con título: «Aspectos Geográficos de la Horticultura de Ciclo Manipulado» y que para el caso que nos ocupa da consistencia a los resultados obtenidos.

Finalmente, en cuanto al calendario de frecuencia de riesgo estimado, se advierte, precisamente, que son los meses de enero y febrero los que provocan la mayor parte de las pérdidas en el área de estudio. Sin embargo, lo anterior no minimiza en absoluto la posibilidad de que acontezca un evento extremo capaz de afectar de forma generalizada en meses como marzo o abril. En esas ocasiones es conveniente estar debidamente preparados, o lo que es lo mismo, reducir el valor de vulnerabilidad por medio de esfuerzos preventivos como los que se aspiran con la utilización de los resultados alcanzados.

\section{BIBLIOGRAFÍA Y FUENTES}

\section{Fuentes}

SIAM [en línea]. [Murcia, España]: Red del Sistema de Información Agrario de Murcia. También disponible en: $<$ http://siam.imida.es/apex/f?p=101:46:174966795856547> [Consulta: 11 mayo 2014]. 
SIAR [en línea]. [Madrid, España]: Sistema de Información Agroclimática para el Regadío del MAGRAMA. También disponible en: < http://eportal.magrama.gob. es/websiar/SeleccionParametrosMap.aspx?dst=1 > [Consulta: 10 mayo 2014].

SIGPAC [en línea]. [Madrid, España]: Sistema de Información Geográfica de Parcelas Agrícolas. También disponible en: <http://sigpac.magrama.es/fega/visor/> [Consulta: 15 mayo 2014].

\section{Bibliografia}

AlONSO SARRÍA, F. (2004): «SIG aplicados al análisis y cartografía de riesgos climáticos. Métodos y Técnicas de Análisis de Riesgos Climáticos», en II Curso de Verano de la Asociación Española de Climatología.

Baeza Gala, F. (1993): «Las heladas y su incidencia económica en la agricultura de Murcia», Papeles de geografía, n. ${ }^{\circ}$ 19, pp. 37-52.

Consejería de Agricultura, Ganadería y Pesca de la Comunidad Autónoma de LA Región de Murcia-Cebas (1992): Riesgo de helada en almendro en la Región de Murcia. Serie estudios. $141 \mathrm{pp}$.

Fernández, C. F., CAstillo, I. P., y Lidón, A. G. (2003): Las heladas en la zona citrícola de la huerta Murciana. Consejería de Agricultura, Agua y Medio Ambiente, Murcia, $95 \mathrm{pp}$.

Fernández-Long, M. E., Müller, G. V., Beltrán-Przekurat, A., y Scarpati, O. E. (2013): «Long-term and recent changes in temperature-based agroclimatic indices in Argentina», International Journal of Climatology, $\mathrm{n}^{\circ} 33$, pp. 1673-1686.

García de Pedraza, L. y García Vega, J. (1991). «Las heladas de irradiación en España», Hojas, N. ${ }^{\circ}$ 1/91, Ministerio de Agricultura Pesca y Alimentación, pp.1-20.

Morales GiL, A. (1997): Aspectos geográficos de la horticultura de ciclo manipulado en España. Alicante, Universidad de Alicante, 167 pp.

Morales Gil, A. (2001): Agua y Territorio en la Región de Murcia. Fundación Centro de Estudios Históricos e Investigaciones Locales Región de Murcia, 270 pp.

Morales Gil, A. (2014). Reflexiones sobre estímulos y carencias actuales de la hortofruticultura española. In Libro jubilar en homenaje al profesor Antonio Gil Olcina. 631-652 pp.

Olcina Cantos, J. (1995): «Riesgos climáticos en las tierras valencianas: incidencia en la actividad agraria», Investigaciones geográficas, $\mathrm{n}^{\circ} 14$, pp. 99-143.

Olcina Cantos, J. y Soriano, F. (2002): «Episodios térmicos extremos. Olas de frío, temporales de nieve y secuencias de helada. Olas de calor», pp. 619-636. En AyalA Carcedo, F.J. y Olcina Cantos, J.; Riesgos naturales. Ariel.

PÉREz Morales, A. (2006): «Tradición, modernización y crisis de la agricultura murciana 1980-2005», en Actas XIII Coloquio de Geografía Rural, Baeza.

QuiÑonero Rubio, J. M., y Alonso SArría, F. (2007): «Creación de Modelos Digitales de Elevaciones a partir de diferentes métodos de interpolación para la determinación de redes de drenaje», en Actas I Jornadas de SIG libre, Girona.

Raso, J. M., Martín Vide, J., y ClaVero, P. (1987): Estadística básica para ciencias sociales. Barcelona, Ariel Geografía, 273 pp.

Snyder, R. L., De Melo-Abreu, J. P., y Matulich, S. (2010): Protección contra las heladas: fundamentos, práctica y economía. Volumen 2, 73-98 pp. 